\title{
INTEGRABILITY AND LIMIT CYCLES OF THE MOON-RAND SYSTEM
}

\author{
LUIS BARREIRA ${ }^{1}$, JAUME LLIBRE ${ }^{2}$ AND CLAUDIA VALLS ${ }^{3}$
}

\begin{abstract}
We study the Darboux integrability of the Moon-Rand polynomial differential system. Moreover we study the limit cycles of the perturbed MoonRand system bifurcating from the equilibrium point located at the origin, when it is perturbed inside the class of all quadratic polynomial differential systems in $\mathbb{R}^{3}$, and we prove that at first order in the perturbation parameter $\varepsilon$ the perturbed system can exhibit one limit cycle, and that at second order it can exhibit four limit cycles bifurcating from the origin. We provide explicit expressions of these limit cycles up to order $O\left(\varepsilon^{2}\right)$.
\end{abstract}

\section{IntRoduCtion AND STATEMENT OF THE MAIN RESUlts}

The Moon-Rand system (introduced by Moon and Rand) was developed to model the control of flexible space structures (see $[11,5,6,10])$. It is a differential system in $\mathbb{R}^{3}$ of the form

$$
\begin{aligned}
& \dot{x}=y, \\
& \dot{y}=-x-x z, \\
& \dot{z}=-\lambda z+\sum_{i=0}^{2} c_{i} x^{i} y^{2-i},
\end{aligned}
$$

where $(x, y, z) \in \mathbb{R}^{3}$ are the variables, $\lambda>0, c_{i}$ are real parameters and the dot indicates derivative with respect to the time $t$. In [10] Mahdi, Romanovski and Shafer studied the Hopf bifurcation of the equilibrium point located at the origin of system (1) using the reduction to the center manifold and studying the Hopf bifurcation on this surface. They found that 2 limit cycles can bifurcate from the origin of system (1). In this paper we study the case when $\lambda=0$, i.e.

$$
\begin{aligned}
& \dot{x}=y, \\
& \dot{y}=-x-x z, \\
& \dot{z}=\alpha x^{2}+\beta x y+\gamma y^{2},
\end{aligned}
$$

where $(x, y, z) \in \mathbb{R}^{3}$ are the state variables and $(\alpha, \beta, \gamma)$ are real parameters. We emphasize that the center manifold is now $\mathbb{R}^{3}$.

We note that system (2) is a family of quadratic systems in a three-dimensional space. Quadratic systems in $\mathbb{R}^{3}$ are the simplest systems after the linear ones. Examples of such systems are the well-known Lorenz system, Rössler system and Rikitake system, among others. These have been investigated in the last decades from

2010 Mathematics Subject Classification. Primary 34C05, 34A34, 34C14.

Key words and phrases. Darboux first integrals, Darboux polynomials, exponential factors, limit cycles, averaging theory. 
different dynamical points of view. Despite their simplicity, quadratic polynomial differential systems are far from being completely understood from the viewpoints of their integrability and of their limit cycles.

The first aim of this paper is to study the existence of Darboux first integrals of system (2), using the Darboux theory of integrability (originated in paper [3]). For the present state of this theory see Chapter 8 of [4], the paper [7], and the references quoted in them. We recall that a first integral of Darboux type is a first integral $H$ which is a function of Darboux type (see below (4) for a precise definition).

The vector field associated to system (2) is

$$
\mathbf{X}=y \frac{\partial}{\partial x}-x(1+z) \frac{\partial}{\partial y}+\left(\alpha x^{2}+\beta x y+\gamma y^{2}\right) \frac{\partial}{\partial z} .
$$

Let $U \subset \mathbb{R}^{3}$ be an open set. We say that a nonconstant function $H: U \rightarrow \mathbb{R}$ is a first integral of the polynomial vector field $\mathbf{X}$ on $U$ if $H(x(t), y(t), z(t))$ is constant for all values of $t$ for which a solution $(x(t), y(t), z(t))$ of $\mathbf{X}$ is defined on $U$. Clearly $H$ is a first integral of $\mathbf{X}$ on $U$ if and only if

$$
\mathbf{X} H=y \frac{\partial H}{\partial x}-x(1+z) \frac{\partial H}{\partial y}+\left(\alpha x^{2}+\beta x y+\gamma y^{2}\right) \frac{\partial H}{\partial z}=0
$$

on $U$. We say that system (2) is completely integrable if there exists two functionally independent first integrals $H_{1}$ and $H_{2}$. We recall that two first integrals $H_{1}$ and $\mathrm{H}_{2}$ are functionally independent if their gradients are linearly independents except maybe on a set of zero Lebesgue measure.

We emphasize that the study of the existence of first integrals is a classical problem in the theory of differential systems, because the knowledge of first integrals of a differential system can be very useful in order to understand and simplify the study of the dynamics of the system. Thus their existence or nonexistence can also be viewed as a measure of the complexity of a differential system.

We recall that a first integral is of Darboux type if it is of the form

$$
f_{1}^{\lambda_{1}} \cdots f_{p}^{\lambda_{p}} F_{1}^{\mu_{1}} \cdots F_{q}^{\mu_{q}}
$$

where $f_{1}, \ldots, f_{p}$ are Darboux polynomials (see section 2 for the definition), $F_{1}, \ldots, F_{q}$ are exponential factors (see section 2 for the definition), and $\lambda_{j}, \mu_{k} \in \mathbb{C}$ for all $j$ and $k$.

The functions of the form (4) are called Darboux functions, and they are the base of the Darboux theory of integrability, which studies when these functions are first integrals or integrating factors. In this last case the first integrals associated to integrating factors given by Darboux functions are the Liouvillian first integrals, for more details see $[4,7]$.

The Darboux theory of integrability is essentially an algebraic theory of integrability based on the invariant algebraic hypersurfaces of a polynomial differential system. In fact to every Darboux polynomial there is associated some invariant algebraic hypersurface (see again section 2), and the exponential factors appear when an invariant algebraic surface has multiplicity larger than 1 , for more details see $[2,4,7]$.

Our main result related to the integrability of system (2) is summarized in the next theorem. 
Theorem 1. The following statements hold for the Moon-Rand polynomial differential system (2).

(a) When $\alpha=\gamma=0$, it is completely integrable with the polynomial first integrals:

$$
H_{1}=\beta \frac{x^{2}}{2}-z, \quad H_{2}=\beta \frac{y^{2}}{2}+z+\frac{z^{2}}{2} .
$$

(b) When $\alpha \neq 0$ or $\gamma \neq 0$ it does not have Darboux first integrals.

The proof of Theorem 1 is given in section 3 .

The second aim of this paper is to study the limit cycles bifurcating from the origin $(0,0,0)$ of the Moon-Rand system (2) when we perturbed it inside the class of all quadratic polynomial differential systems in $\mathbb{R}^{3}$. We will use the averaging theory of first and second order. More precisely we consider the system

(5)

$$
\begin{aligned}
\dot{x}= & y+\varepsilon\left(a_{1} x+a_{2} y+a_{3} z+a_{4} x^{2}+a_{5} y x+a_{6} z x+a_{7} y^{2}+a_{8} y z+a_{9} z^{2}\right) \\
& +\varepsilon^{2}\left(a_{10}+a_{11} x+a_{12} y+a_{13} z+a_{14} x^{2}+a_{15} y x+a_{16} z x+a_{17} y^{2}+a_{18} y z\right. \\
& \left.+a_{19} z^{2}\right), \\
\dot{y}= & -x(z+1)+\varepsilon\left(b_{1} x+b_{2} y+b_{3} z+b_{4} x^{2}+b_{5} y x+b_{6} z x+b_{7} y^{2}+b_{8} y z+b_{9} z^{2}\right) \\
& +\varepsilon^{2}\left(b_{10}+b_{11} x+b_{12} y+b_{13} z+b_{14} x^{2}+b_{15} y x+b_{16} z x+b_{17} y^{2}+b_{18} y z\right. \\
& \left.+b_{19} z^{2}\right), \\
\dot{z}= & \alpha x^{2}+\beta x y+\gamma y^{2}+\varepsilon\left(c_{1} x+c_{2} y+c_{3} z+c_{4} x^{2}+c_{5} y x+c_{6} z x+c_{7} y^{2}+c_{8} y z\right. \\
& \left.+c_{9} z^{2}\right)+\varepsilon^{2}\left(c_{10}+c_{11} x+c_{12} y+c_{13} z+c_{14} x^{2}+c_{15} y x+c_{16} z x+c_{17} y^{2}\right. \\
& \left.+c_{18} y z+c_{19} z^{2}\right) .
\end{aligned}
$$

Assuming that $a_{6}+b_{8} \neq 0$ we introduce the notation

$$
S_{13}=\left(b_{3} c_{1}+c_{13}-a_{3} c_{2}\right)^{2}+2 c_{9}\left(a_{10} c_{2}-b_{10} c_{1}\right),
$$

and

(7)

$$
\begin{aligned}
A= & -\frac{\left(2 a_{6}+2 b_{8}+c_{9}\right) \alpha^{2}}{8\left(a_{6}+b_{8}\right)^{2}} \\
B= & -\frac{1}{2\left(a_{6}+b_{8}\right)^{2}}\left(2\left(c_{4}+c_{7}+\left(a_{2}+b_{1}\right) \alpha-a_{1} \beta\right) a_{6}^{2}+\left(2\left(a_{11}+b_{12}\right) \alpha\right.\right. \\
& \left.-\left(3 b_{3} c_{1}+c_{13}-3 a_{3} c_{2}\right) \alpha+4 b_{8}\left(c_{4}+c_{7}+\left(a_{2}+b_{1}\right) \alpha-a_{1} \beta\right)\right) a_{6}+ \\
& b_{8}\left(2 a_{11}+2 b_{12}-3 b_{3} c_{1}-c_{13}+3 a_{3} c_{2}\right) \alpha+2\left(a_{11}+b_{12}-b_{3} c_{1}+a_{3} c_{2}\right) c_{9} \alpha \\
& \left.+2 b_{8}^{2}\left(c_{4}+c_{7}+\left(a_{2}+b_{1}\right) \alpha-a_{1} \beta\right)\right), \\
C= & \frac{1}{\left(a_{6}+b_{8}\right)^{2}}\left(\left(a_{10} c_{2}-b_{10} c_{1}\right) a_{6}^{2}+2\left(-a_{3}^{2} c_{2}^{2}+\left(a_{10} b_{8}+a_{3}\left(2 b_{3} c_{1}+c_{13}\right)\right) c_{2}\right.\right. \\
& -c_{1}\left(b_{10} b_{8}+b_{3}\left(b_{3} c_{1}+c_{13}\right)\right)+a_{11}\left(b_{3} c_{1}+c_{13}-a_{3} c_{2}\right)+ \\
& \left.b_{12}\left(b_{3} c_{1}+c_{13}-a_{3} c_{2}\right)\right) a_{6}+b_{8}\left(-2 a_{3}^{2} c_{2}^{2}+\left(a_{10} b_{8}+2 a_{3}\left(2 b_{3} c_{1}+c_{13}\right)\right) c_{2}\right. \\
& -c_{1}\left(b_{10} b_{8}+2 b_{3}\left(b_{3} c_{1}+c_{13}\right)\right)+2 a_{11}\left(b_{3} c_{1}+c_{13}-a_{3} c_{2}\right) \\
& \left.\left.+2 b_{12}\left(b_{3} c_{1}+c_{13}-a_{3} c_{2}\right)\right)-2\left(a_{11}+b_{12}-b_{3} c_{1}+a_{3} c_{2}\right)^{2} c_{9}\right) .
\end{aligned}
$$


Theorem 2. The following statements hold for system (5).

(a) Using the averaging theory of first order, for $\varepsilon \neq 0$ sufficiently small system (5) has a limit cycle bifurcating from the equilibrium point at the origin when $\varepsilon=0$ and $c_{3} c_{10}\left(a_{1}+a_{2}\right) \neq 0$. This limit cycle is of the form $(x(t, \varepsilon), y(t, \varepsilon), z(t, \varepsilon))$ where

$$
x(t, \varepsilon)=O\left(\varepsilon^{2}\right), \quad y(t, \varepsilon)=O\left(\varepsilon^{2}\right), \quad z(t, \varepsilon)=-\varepsilon \frac{c_{10}}{c_{3}} z+O\left(\varepsilon^{2}\right) .
$$

(b) Using the averaging theory of second order, for $\varepsilon \neq 0$ sufficiently small system (5) has four limit cycles bifurcating from the equilibrium point at the origin when $\varepsilon=0, b_{2}=-a_{1}, c_{3}=c_{10}=0, \gamma=-\alpha, a_{6}+b_{8} \neq 0$ and $A \neq 0$. Two limit cycles are of the form $(x(t, \varepsilon), y(t, \varepsilon), z(t, \varepsilon))$ where

$x(t, \varepsilon)=O\left(\varepsilon^{2}\right), \quad y(t, \varepsilon)=O\left(\varepsilon^{2}\right), \quad z(t, \varepsilon)=\varepsilon \frac{-b_{3} c_{1}-c_{13}+a_{3} c_{2} \pm \sqrt{S_{13}}}{2 c_{9}} z+O\left(\varepsilon^{2}\right)$, whenever $S_{13}>0$ and $c_{9} d_{1,2} \neq 0$ (see the proof of Theorem 2 for the definition of $\left.d_{1,2}\right)$.

The other two limit cycles are of the form $(x(t, \varepsilon), y(t, \varepsilon), z(t, \varepsilon))$ where

$$
\begin{aligned}
& x(t, \varepsilon)=\varepsilon \sqrt{-\frac{B}{2 A} \pm \frac{\sqrt{B^{2}-4 A C}}{2 A}} \cos t+O\left(\varepsilon^{2}\right), \\
& y(t, \varepsilon)=\varepsilon \sqrt{-\frac{B}{2 A} \pm \frac{\sqrt{B^{2}-4 A C}}{2 A}} \sin t+O\left(\varepsilon^{2}\right), \\
& z(t, \varepsilon)=\varepsilon \frac{\left(B \mp \sqrt{B^{2}-4 A C}\right) \alpha-8 A\left(a_{11}+b_{12}-b_{3} c_{1}+a_{3} c_{2}\right)}{8 A\left(a_{6}+b_{8}\right)} z+O\left(\varepsilon^{2}\right),
\end{aligned}
$$

whenever

$$
C \neq 0,-\frac{B}{2 A} \pm \frac{\sqrt{B^{2}-4 A C}}{2 A}>0, d_{3,4} \neq 0
$$

(see the proof of Theorem 2 for the definition of $d_{3,4}$ ).

The proof of Theorem 2 is given in section 4 . It uses the averaging theory described in Theorem 11 of the appendix.

\section{BASIC RESULTS}

Let $h=h(x, y, z) \in \mathbb{C}[x, y, z] \backslash \mathbb{C}$. As usual $\mathbb{C}[x, y, z]$ denotes the ring of all complex polynomials in the variables $x, y, z$. We say that $h$ is a Darboux polynomial of system (2) if it satisfies

$$
\mathbf{X} h=K h
$$

for some polynomial $K=K(x, y, z) \in \mathbb{C}[x, y, z]$, called the cofactor of $h$, of degree at most 1. Every Darboux polynomial $h$ defines an invariant algebraic surface $h=0$, i.e., if a trajectory of system (2) has a point in $h=0$, then the whole trajectory is contained in $h=0$, see [4] for more details. When $K=0$ the Darboux polynomial $h$ is a polynomial first integral.

We note that we will look for Darboux polynomials in $\mathbb{C}$ instead of $\mathbb{R}$. This is due to the fact that real first integrals can come from complex Darboux polynomials. The reason for this is the following: if there exists a complex Darboux polynomial of 
a real polynomial differential system then its conjugate is also a Darboux polynomial and their product is a real Darboux polynomial.

We recall the following auxiliary result that was proved in [4].

Lemma 3. Let $f$ be a polynomial and let $f=\prod_{j=1}^{s} f_{j}^{\alpha_{j}}$ be its decomposition into irreducible factors in $\mathbb{C}[x, y, z]$. Then $f$ is a Darboux polynomial if and only if each $f_{j}$ is a Darboux polynomial. Moreover, if $K$ and $K_{j}$ are the cofactors of $f$ and $f_{j}$, then $K=\sum_{j=1}^{s} \alpha_{j} K_{j}$.

An exponential factor $E$ of system (2) is a function of the form $E=\exp (g / h) \notin \mathbb{C}$ with $g, h \in \mathbb{C}[x, y, z]$ satisfying $(g, h)=1$ and

$$
\mathbf{X} E=L E,
$$

for some polynomial $L=L(x, y, z)$ of degree at most one, called the cofactor of $E$.

A geometrical meaning of the notion of exponential factor is given by the next result.

Proposition 4. If $E=\exp (g / h)$ is an exponential factor of the polynomial differential system (2) and $h$ is a nonconstant polynomial, then $h=0$ is an invariant algebraic surface, and eventually $e^{g}$ can be an exponential factor, coming from the multiplicity of the infinite invariant plane.

The proof of Proposition 4 can be found in $[2,8]$. We explain a little the last part of the statement of Proposition 4. If we extend to the projective space $\mathbb{P R}^{3}$ the polynomial differential system (2) defined in the affine space $\mathbb{R}^{3}$, then the plane at infinity is always invariant by the flow of the extended differential system. Moreover, if this invariant plane has multiplicity higher than 1 , then it creates exponential factors of the form $e^{g}$, see [8] for more details.

We shall use the following result.

Theorem 5. Suppose that the polynomial vector field $\mathbf{X}$ of degree $m$ defined in $\mathbb{C}^{3}$ admits $p$ invariant algebraic surfaces $f_{i}=0$ with cofactors $K_{i}$, for $i=1, \ldots, p$ and $q$ exponential factors $E_{j}=\exp \left(g_{j} / h_{j}\right)$ with cofactors $L_{j}$, for $j=1, \ldots, q$. Then there exist $\lambda_{i}, \mu_{j} \in \mathbb{C}$ not all zero such that

$$
\sum_{i=1}^{p} \lambda_{i} K_{i}+\sum_{j=1}^{q} \mu_{j} L_{j}=0
$$

if and only if the function of Darboux type

$$
f_{1}^{\lambda_{1}} \cdots f_{p}^{\lambda_{p}} E_{1}^{\mu_{1}} \cdots E_{q}^{\mu_{q}}
$$

is a first integral of $\mathbf{X}$.

Theorem 5 is proved in $[4,8]$.

Lemma 6. Assume that $\exp \left(g_{1} / h_{1}\right), \ldots, \exp \left(g_{r} / h_{r}\right)$ are exponential factors of a polynomial differential system

$$
x^{\prime}=P(x, y, z), y^{\prime}=Q(x, y, z), z^{\prime}=R(x, y, z),
$$


for some $P, Q, R \in \mathbb{C}[x, y, z]$, with cofactors $L_{j}$ for $j=1, \ldots, r$. Then

$$
\exp (G)=\exp \left(g_{1} / h_{1}+\cdots+g_{r} / h_{r}\right)
$$

is also an exponential factor of system (8), with cofactor $L=\sum_{j=1}^{r} L_{j}$.

Proof. Using that for $j=1, \ldots, r, E_{1}=\exp \left(g_{1} / h_{1}\right), \ldots, E_{r}=\exp \left(g_{r} / h_{r}\right)$ are exponential factors of system (8) with cofactors $L_{j}$ we have

$$
\frac{\partial\left(g_{j} / h_{j}\right)}{\partial x} P(x, y, z) E_{j}+\frac{\partial\left(g_{j} / h_{j}\right)}{\partial y} Q(x, y, z) E_{j}+\frac{\partial\left(g_{j} / h_{j}\right)}{\partial z} R(x, y, z) E_{j}=L_{j} E_{j},
$$

or equivalently

$$
\frac{\partial\left(g_{j} / h_{j}\right)}{\partial x} P(x, y, z)+\frac{\partial\left(g_{j} / h_{j}\right)}{\partial y} Q(x, y, z)+\frac{\partial\left(g_{j} / h_{j}\right)}{\partial z} R(x, y, z)=L_{j} .
$$

Therefore if we set $G=\sum_{j=1}^{r} g_{j} / h_{j}$ we get that

$$
\frac{\partial G}{\partial x} P(x, y, z)+\frac{\partial G}{\partial y} Q(x, y, z)+\frac{\partial G}{\partial z} R(x, y, z)=\sum_{j=1}^{r} L_{j}=L,
$$

and thus if $E=\exp (G)$ we obtain

$$
\frac{\partial G}{\partial x} P(x, y, z) E+\frac{\partial G}{\partial y} Q(x, y, z) E+\frac{\partial G}{\partial z} R(x, y, z) E=L E .
$$

This concludes the proof of the lemma.

\section{Proof of Theorem 1.}

When $\gamma=\alpha=0$ the result follows directly checking condition (3). This proves statement (a).

Now we shall prove statement (b). We assume that $\gamma \neq 0$ or $\alpha \neq 0$.

We consider the automorphism $\tau: \mathbb{C}^{3}[x, y, z] \rightarrow \mathbb{C}^{3}[x, y, z]$ defined by

$$
\tau(x, y, x)=\tau(-x,-y, z) .
$$

Proposition 7. If $g$ is an irreducible Darboux polynomial of system (2) with cofactor

$$
K=\alpha_{0}+\alpha_{1} x+\alpha_{2} y+\alpha_{3} z
$$

then $f=g \cdot \tau g$ is a Darboux polynomial invariant by $\tau$ with a cofactor of the form

$$
K_{f}=2 \alpha_{0}+2 \alpha_{3} z .
$$

Proof. Since system (2) is invariant by $\tau$, the function $\tau g$ is a Darboux polynomial of system (2) with cofactor $\tau(K)$. Moreover, by Lemma $3, g \cdot \tau g$ is also a Darboux polynomial of system (2) with cofactor $K+\tau(K)$. Therefore again by Lemma 3, the cofactor of $f$ is $K_{f}=K+\tau(K)$ which is given in (9).

Proposition 8. Let $f$ be a Darboux polynomial of system (2) invariant by $\tau$ with nonzero cofactor $K_{f}$. Then $K_{f}=2 \alpha_{0}$.

Proof. Let $f=f(x, y, z)$ be a Darboux polynomial of system (2) with nonzero cofactor $K_{f}$. Then it satisfies

$$
y \frac{\partial f}{\partial x}-x(1+z) \frac{\partial f}{\partial y}+\left(\alpha x^{2}+\beta x y+\gamma y^{2}\right) \frac{\partial f}{\partial z}=2\left(\alpha_{0}+\alpha_{3} z\right) f .
$$


We decompose $f$ into its homogeneous parts as

$$
f=\sum_{j=0}^{n} f_{j}(x, y, z)
$$

where each $f_{j}$ is a homogeneous polynomial of degree $j$. Computing the terms of degree $n+1$ in (10), we get

$$
-x z \frac{\partial f_{n}}{\partial y}+\left(\alpha x^{2}+\beta x y+\gamma y^{2}\right) \frac{\partial f_{n}}{\partial z}=2 \alpha_{3} z f_{n} .
$$

Solving it we get

$$
f_{n}=G_{n}\left(x, 3 x\left(\beta y^{2}+z^{2}\right)+2 y\left(3 \alpha x^{2}+\gamma y^{2}\right)\right) e^{-\frac{2 \alpha_{3} y}{x}},
$$

where $G_{n}$ is any function in the variables $x$ and $3 x\left(\beta y^{2}+z^{2}\right)+2 y\left(3 \alpha x^{2}+\gamma y^{2}\right)$. Since $f_{n}$ must be a polynomial we must have $\alpha_{3}=0$.

The following result characterizes the Darboux polynomials of system (2) that are invariant by $\tau$ and have nonzero cofactor.

Proposition 9. There are no Darboux polynomial of system (2) invariant by $\tau$ and with nonzero cofactor.

Proof. Let $f=f(x, y, z)$ be a Darboux polynomial of system (2) invariant by $\tau$ with nonzero cofactor $K=2 \alpha_{0}$.

In order to simplify the computations we do the rescaling

$$
x=\mu^{-2} X, \quad y=\mu^{-2} Y, \quad z=\mu^{-1} Z, \quad t=\mu^{3} T,
$$

with $\mu \in \mathbb{R} \backslash\{0\}$. Then system (2) becomes

$$
\begin{aligned}
& X^{\prime}=\mu^{3} Y, \\
& Y^{\prime}=-\mu^{2} X Z-\mu^{3} X, \\
& Z^{\prime}=\alpha X^{2}+\beta X Y+\gamma Y^{2},
\end{aligned}
$$

where the prime denotes the derivative with respect to the variable $T$. Now we define

$$
F(\mu, X, Y, Z)=\mu^{m} f\left(\mu^{-2} X, \mu^{-2} Y, \mu^{-1} Z\right)
$$

where $m$ is the largest integer such that $\mu^{-m}$ appears in $f\left(\mu^{-2} X, \mu^{-2} Y, \mu^{-1} Z\right)$. We write

$$
F(\mu, X, Y, Z)=\sum_{i=0}^{m} \mu^{i} F_{i}(X, Y, Z)
$$


where $F_{i}$ is the coefficient of $\mu^{i}$ in the polynomial $F(\mu, X, Y, Z)$. Since $F(\mu, X, Y, Z)$ is a Darboux polynomial of system (11), equation (2) can be written as

$$
\begin{aligned}
& \mu^{3} Y \frac{\partial}{\partial X}\left(\sum_{i=0}^{m} \mu^{i} F_{i}(X, Y, Z)\right)-\mu^{2}(X Z+\mu X) \frac{\partial}{\partial Y}\left(\sum_{i=0}^{m} \mu^{i} F_{i}(X, Y, Z)\right) \\
& +\left(\alpha X^{2}+\beta X Y+\gamma Y^{2}\right) \frac{\partial}{\partial Z}\left(\sum_{i=0}^{m} \mu^{i} F_{i}(X, Y, Z)\right) \\
& =2 \alpha_{0}\left(\sum_{i=0}^{m} \mu^{i} F_{i}(X, Y, Z)\right) .
\end{aligned}
$$

Computing the coefficient of $\mu^{0}$ in (12) we get

$\left(\alpha X^{2}+\beta X Y+\gamma Y^{2}\right) \frac{\partial F_{0}}{\partial Z}=2 \alpha_{0} F_{0}, \quad$ that is $\quad F_{0}=G_{0}(X, Y) e^{2 \alpha_{0} Z /\left(\alpha X^{2}+\beta X Y+\gamma Y^{2}\right)}$, where $G_{0}$ is any function in the variables $X, Y$. Since $F_{0}$ must be a polynomial we must have $\alpha_{0}=0$. This concludes the proof.

Proposition 10. There are no polynomial first integrals of system (2) invariant by $\tau$.

Proof. Let $f=f(x, y, z)$ be a polynomial first integral of system (2) invariant by $\tau$.

In order to simplify the computations we do the rescaling

$$
x=\mu X, \quad y=\mu Y, \quad z=Z, \quad t=T,
$$

with $\mu \in \mathbb{R} \backslash\{0\}$. Then system (2) becomes

$$
\begin{aligned}
& X^{\prime}=Y, \\
& Y^{\prime}=-X-X Z, \\
& Z^{\prime}=\mu^{2}\left(\alpha X^{2}+\beta X Y+\gamma Y^{2}\right),
\end{aligned}
$$

where the prime denotes the derivative with respect to the variable $T$. Now we define

$$
F(\mu, X, Y, Z)=f(\mu X, \mu Y, Z)
$$

and we write

$$
F(\mu, X, Y, Z)=\sum_{i=0}^{m} \mu^{i} F_{i}(X, Y, Z),
$$

where $F_{i}$ is the coefficient of $\mu^{i}$ in the polynomial $F(\mu, X, Y, Z)$. Since $F(\mu, X, Y, Z)$ is a Darboux polynomial of system (13) with zero cofactor, we have

$$
\begin{aligned}
& Y \frac{\partial}{\partial X}\left(\sum_{i=0}^{m} \mu^{i} F_{i}(X, Y, Z)\right)-(X+X Z) \frac{\partial}{\partial Y}\left(\sum_{i=0}^{m} \mu^{i} F_{i}(X, Y, Z)\right) \\
& +\mu^{2}\left(\alpha X^{2}+\beta X Y+\gamma Y^{2}\right) \frac{\partial}{\partial Z}\left(\sum_{i=0}^{m} \mu^{i} F_{i}(X, Y, Z)\right)=0 .
\end{aligned}
$$

Computing the coefficient of $\mu^{0}$ in (14) we get $Y \frac{\partial F_{0}}{\partial X}-(X+X Z) \frac{\partial F_{0}}{\partial Y}=0$ that is $F_{0}=G_{0}\left(Z, H_{2}\right)$, where $H_{2}=X^{2}(1+Z)+Y^{2}$, 
where $G_{0}$ is any function in the variables $Z, H_{2}$. Since $F_{0}$ is the coefficient of $\mu^{0}$ in (14), $F_{0}=F_{0}(Z)$.

Computing the coefficient of $\mu$ in (14) we get again that $F_{1}=F_{1}\left(Z, H_{2}\right)$, where $G_{1}$ is any function in the variables $Z, H_{2}$. Since $F_{1}$ must be linear in the variables $X$ and $Y$, it follows that $F_{1}=0$.

Computing the coefficient of $\mu^{2}$ in (14) we obtain

$$
Y \frac{\partial F_{2}}{\partial X}-(X+X Z) \frac{\partial F_{2}}{\partial Y}=\left(\alpha X^{2}+\beta X Y+\gamma Y^{2}\right) F_{0}^{\prime}(Z)
$$

Solving this linear partial differential equation we get

$$
\begin{aligned}
F_{2} & =G_{2}\left(Z, H_{2}\right)-\frac{X(Y \alpha+(Z+1)(X \beta-Y \gamma))}{2(-Z-1)} \\
& +\frac{\left((Z+1) X^{2}+Y^{2}\right)(\alpha+Z \gamma+\gamma) \log (Y \sqrt{-Z-1}-X(Z+1)) F_{0}^{\prime}(Z)}{2(-Z-1)^{3 / 2}}
\end{aligned}
$$

where $G_{2}$ is any function in the variables $Z, H_{2}$. Since $F_{2}$ must be a polynomial we must have $(\alpha+\gamma+\gamma Z) F_{0}^{\prime}(Z)=0$. If $\alpha=0$ then $\gamma \neq 0$ and $F_{0}^{\prime}(Z)=0$, and if $\gamma=0$ then $\alpha \neq 0$ and again $F_{0}^{\prime}(Z)=0$. In short $F_{0}^{\prime}(Z)=0$, and we can assume that $F_{0}=0$.

Now we proceed by induction. Assume that $F_{k}=0$ for $k=0, \ldots, \ell-1$ with $\ell \geq 1$ and we shall prove it for $k=\ell$.

By induction hypothesis, the coefficient of $\mu^{\ell}$ in (14) satisfies

$$
Y \frac{\partial F_{\ell}}{\partial X}-(X+X Z) \frac{\partial F_{\ell}}{\partial Y}=0 \text { that is } F_{\ell}=G_{\ell}\left(Z, H_{2}\right)
$$

where $G_{\ell}$ is any function in the variables $Z, H_{2}$. Since $F_{\ell}$ must be a homogeneous polynomial of degree $\ell$ in the variables $X$ and $Y$, thinking of $Z$ as a constant, we get that if $\ell$ is odd then $F_{\ell}=0$ because $H_{2}$ is of even degree in the variables $X$ and $Y$, and we are done. If $\ell$ is even then $F_{\ell}=M_{\ell}(Z) H_{2}^{\ell / 2}$, where $M_{\ell}$ is a polynomial in the variable $Z$.

Now proceeding as in the case $F_{1}$ we obtain that $F_{\ell+1}=0$. Computing the coefficient of $\mu^{\ell+2}$ in (14) we get

$$
\begin{aligned}
& Y \frac{\partial F_{\ell+2}}{\partial X}-(X+X Z) \frac{\partial F_{\ell+2}}{\partial Y}=\left(\alpha X^{2}+\beta X Y+\gamma Y^{2}\right) F_{\ell}^{\prime}(Z) \\
& =\left(\alpha X^{2}+\beta X Y+\gamma Y^{2}\right)\left(H_{2}^{\ell / 2} M_{\ell}^{\prime}(Z)+\frac{\ell}{2} X^{2} H_{2}^{\ell / 2-1} M_{\ell}(Z)\right)
\end{aligned}
$$


Solving this linear partial differential equation we obtain

$$
\begin{aligned}
F_{\ell+2}= & \frac{1}{16(z+1)^{2}\left((z+1) x^{2}+y^{2}\right)}\left(\ell x \left(2(z+1)^{2} \beta x^{3}-y(z+1)(-5 \alpha+z \gamma+\gamma) x^{2}\right.\right. \\
& \left.+y^{3}(3 \alpha+z \gamma+\gamma)\right) M(z)\left((z+1) x^{2}+y^{2}\right)^{\ell / 2}+8(z+1)\left(x \left((z+1)^{2} \beta x^{3}\right.\right. \\
& \left.-y(z+1)(-\alpha+z \gamma+\gamma) x^{2}+y^{2}(z+1) \beta x+y^{3}(\alpha-(z+1) \gamma)\right) M^{\prime}(z) \\
& \left.\left.\left((z+1) x^{2}+y^{2}\right)^{\ell / 2}+2(z+1) G_{\ell+2}\left(z, H_{2}\right)\left((z+1) x^{2}+y^{2}\right)\right)\right) \\
& -\frac{1}{16(-z-1)^{5 / 2}}\left(\left((z+1) x^{2}+y^{2}\right)^{(\ell+2) / 2} \log (y \sqrt{-z-1}-x(z+1))\right. \\
& \left.\left(\ell(3 \alpha+z \gamma+\gamma) M(z)+8(z+1)(\alpha+z \gamma+\gamma) M^{\prime}(z)\right)\right)
\end{aligned}
$$

where $G_{\ell+2}$ is any function in the variables $Z, H_{2}$. Since $F_{\ell+2}$ must be a polynomial we must have

$$
8(1+Z)(\gamma+\alpha+\alpha Z) M_{\ell}^{\prime}(Z)+\frac{\ell}{32}(3 \alpha+\gamma+\gamma Z) M_{\ell}(Z)=0 .
$$

Solving this linear differential equation in $M_{\ell}(Z)$ we get

$$
M_{\ell}(Z)=C_{\ell}(1+Z)^{-3 \ell / 8}(\alpha+\gamma+\gamma Z)^{\ell / 4}, \quad C_{\ell} \in \mathbb{C} .
$$

Since $M_{\ell}$ must be a polynomial, $\ell>0$ and either $\alpha \neq 0$ or $\gamma \neq 0$ we get that $C_{\ell}=0$, i.e. $M_{\ell}(Z)=0$. Then since $F_{\ell}=M_{\ell}(Z) H_{2}^{\ell / 2}$, we obtain that $F_{\ell}=0$ and this concludes the proof of the lemma.

In summary, from Propositions 7, 9 and 10 it follows that system (2) has no Darboux polynomials.

Now it follows from Proposition 4 that in order to have a first integral of Darboux type we must have $q$ exponential factors $E_{j}=\exp \left(g_{j}\right)$ with cofactors $L_{j}$ such that $\sum_{j=1}^{q} \mu_{j} L_{j}=0$. Let $H=\sum_{j=1}^{q} \mu_{j} g_{j}$, then by Lemma $6 E=\exp (H)$ is an exponential factor of system (2) with cofactor $L=\sum_{j=1}^{q} \mu_{j} L_{j}=0$. Therefore $\exp (H)$ is a first integral because its cofactor is zero. So $H$ is a polynomial first integral of system (2), but by the argument used in the proof of Proposition 10 this is not possible. This completes the proof of statement (b) of Theorem 1.

\section{Proof of Theorem 2}

First in order to write the differential system (5) into the normal form for applying the averaging theory described in the appendix we do the change of variables

$$
x=\varepsilon R \cos \theta, \quad y=\varepsilon R \sin \theta, \quad z=\varepsilon Z .
$$

Recall that this is a change of variables when $R>0$. Moreover doing this change of variables the angular variable $\theta$ appears in the system. Later on the variable $\theta$ will be used for obtaining the periodicity necessary for applying the averaging theory. So now we consider the system $(\dot{R}, \dot{\theta}, \dot{Z})$. 
We take as the new time the variable $\theta$, and consequently the previous system in $(\dot{R}, \dot{\theta}, \dot{Z})$ becomes

$$
\begin{aligned}
& R^{\prime}=\frac{d R}{d \theta}=-\varepsilon F_{11}(r, \theta, Z)+\varepsilon^{2} F_{12}(r, \theta, Z)+O\left(\varepsilon^{3}\right), \\
& Z^{\prime}=\frac{d Z}{d \theta}=-\varepsilon F_{21}(r, \theta, Z)+\varepsilon^{2} F_{22}(r, \theta, Z)+O\left(\varepsilon^{3}\right),
\end{aligned}
$$

where

$$
\begin{aligned}
& F_{11}=-\frac{1}{2}\left(a_{1}-b_{2}\right) R \cos ^{2} \theta-\left(a_{10}+a_{3} Z\right) \cos \theta-R\left(a_{2}+b_{1}-Z\right) \sin \theta \cos \theta \\
& +\frac{1}{2}\left(a_{1}-b_{2}\right) R \sin ^{2} \theta-\frac{1}{2}\left(a_{1}+b_{2}\right) R-\left(b_{10}+b_{3} Z\right) \sin \theta, \\
& F_{12}=-\frac{1}{2}(\alpha-\gamma) \cos ^{2} \theta R^{2}+\frac{1}{2}(\alpha-\gamma) \sin ^{2} \theta R^{2}-\frac{1}{2}(\alpha+\gamma) R^{2}-\beta \cos \theta \sin \theta R^{2} \\
& -c_{1} \cos \theta R-c_{2} \sin \theta R-c_{10}-c_{3} Z \text {, } \\
& F_{21}=-\frac{1}{4}\left(a_{1}-b_{2}\right) R\left(a_{2}+b_{1}-Z\right) \cos ^{4} \theta-\frac{1}{4}\left(a_{4} R^{2}-a_{7} R^{2}-b_{5} R^{2}-2 a_{3} Z^{2}\right. \\
& \left.-2 a_{10} Z+2 a_{2} a_{3} Z+2 a_{3} b_{1} Z+2 a_{1} b_{3} Z-2 b_{2} b_{3} Z\right) \cos ^{3} \theta \\
& +\frac{1}{2} R\left(a_{1}+a_{2}+b_{1}-b_{2}-Z\right)\left(a_{1}-a_{2}-b_{1}-b_{2}+Z\right) \sin \theta \cos ^{3} \theta \\
& +\frac{3}{2}\left(a_{1}-b_{2}\right) R\left(a_{2}+b_{1}-Z\right) \sin ^{2} \theta \cos ^{2} \theta-\frac{1}{2 R}\left(a_{11} R^{2}+a_{1} b_{1} R^{2}-b_{12} R^{2}\right. \\
& \left.+a_{2} b_{2} R^{2}-a_{1} Z R^{2}+a_{6} Z R^{2}-b_{8} Z R^{2}+2 a_{3} b_{3} Z^{2}\right) \cos ^{2} \theta \\
& -\frac{3}{4}\left(a_{5} R^{2}+b_{4} R^{2}-b_{7} R^{2}-2 b_{3} Z^{2}-2 a_{1} a_{3} Z-2 b_{10} Z+2 a_{3} b_{2} Z\right. \\
& \left.+2 a_{2} b_{3} Z+2 b_{1} b_{3} Z\right) \sin \theta \cos ^{2} \theta-\frac{1}{2} R\left(a_{1}+a_{2}+b_{1}-b_{2}-Z\right) \\
& \left(a_{1}-a_{2}-b_{1}-b_{2}+Z\right) \sin ^{3} \theta \cos \theta+\frac{3}{4}\left(a_{4} R^{2}-a_{7} R^{2}-b_{5} R^{2}-2 a_{3} Z^{2}\right. \\
& \left.-2 a_{10} Z+2 a_{2} a_{3} Z+2 a_{3} b_{1} Z+2 a_{1} b_{3} Z-2 b_{2} b_{3} Z\right) \sin ^{2} \theta \cos \theta \\
& -\frac{1}{4}\left(3 a_{4} R^{2}+a_{7} R^{2}+b_{5} R^{2}-2 a_{3} Z^{2}+4 a_{9} Z^{2}-2 a_{10} Z+4 a_{13} Z\right. \\
& \left.-2 a_{2} a_{3} Z+2 a_{3} b_{1} Z+2 a_{1} b_{3} Z+2 b_{2} b_{3} Z\right) \cos \theta+\frac{1}{2 R}\left(a_{1}^{2} R^{2}+a_{2}^{2} R^{2}\right. \\
& -b_{1}^{2} R^{2}-b_{2}^{2} R^{2}-Z^{2} R^{2}-2 a_{12} R^{2}-2 b_{11} R^{2}-2 a_{8} Z R^{2}+2 b_{1} Z R^{2} \\
& \left.-2 b_{6} Z R^{2}+2 a_{3}^{2} Z^{2}-2 b_{3}^{2} Z^{2}\right) \sin \theta \cos \theta-\frac{1}{4}\left(a_{1}-b_{2}\right) R\left(a_{2}+b_{1}-Z\right) \sin ^{4} \theta \\
& +\frac{1}{4}\left(a_{5} R^{2}+b_{4} R^{2}-b_{7} R^{2}-2 b_{3} Z^{2}-2 a_{1} a_{3} Z-2 b_{10} Z+2 a_{3} b_{2} Z\right. \\
& \left.+2 a_{2} b_{3} Z+2 b_{1} b_{3} Z\right) \sin ^{3} \theta+\frac{1}{2 R}\left(a_{11} R^{2}+a_{1} b_{1} R^{2}-b_{12} R^{2}+a_{2} b_{2} R^{2}\right. \\
& \left.-a_{1} Z R^{2}+a_{6} Z R^{2}-b_{8} Z R^{2}+2 a_{3} b_{3} Z^{2}\right) \sin ^{2} \theta-\frac{1}{4}\left(2 a_{11}-a_{1} a_{2}+a_{1} b_{1}\right.
\end{aligned}
$$




$$
\begin{aligned}
& \left.+2 b_{12}-a_{2} b_{2}+b_{1} b_{2}\right) R+\frac{1}{4}\left(a_{1}-2 a_{6}+b_{2}-2 b_{8}\right) R Z-\frac{1}{4}\left(a_{5} R^{2}+b_{4} R^{2}\right. \\
& +3 b_{7} R^{2}-2 b_{3} Z^{2}+4 b_{9} Z^{2}-2 a_{1} a_{3} Z-2 b_{10} Z+4 b_{13} Z-2 a_{3} b_{2} Z \\
& \left.-2 a_{2} b_{3} Z+2 b_{1} b_{3} Z\right) \sin \theta, \\
& F_{22}=-\frac{1}{8} R^{2}\left(a_{2} \alpha+b_{1} \alpha-Z \alpha+a_{1} \beta-b_{2} \beta-a_{2} \gamma-b_{1} \gamma+Z \gamma\right) \cos ^{4} \theta \\
& -\frac{1}{4} R\left(a_{2} c_{1}+b_{1} c_{1}-Z c_{1}+a_{1} c_{2}-b_{2} c_{2}+b_{10} \alpha+b_{3} Z \alpha+a_{10} \beta\right. \\
& \left.+a_{3} Z \beta-b_{10} \gamma-b_{3} Z \gamma\right) \cos ^{3} \theta+\frac{1}{2} R^{2}\left(a_{1} \alpha-b_{2} \alpha-a_{2} \beta-b_{1} \beta\right. \\
& \left.+Z \beta-a_{1} \gamma+b_{2} \gamma\right) \sin \theta \cos ^{3} \theta+\frac{3}{4} R^{2}\left(a_{2} \alpha+b_{1} \alpha-Z \alpha+a_{1} \beta\right. \\
& \left.-b_{2} \beta-a_{2} \gamma-b_{1} \gamma+Z \gamma\right) \sin ^{2} \theta \cos ^{2} \theta-\frac{1}{2}\left(c_{4} R^{2}-c_{7} R^{2}+\right. \\
& b_{1} \alpha R^{2}-Z \alpha R^{2}+a_{2} \gamma R^{2}-c_{3} Z^{2}+b_{3} c_{1} Z-c_{10} Z+a_{3} c_{2} Z \\
& \left.+a_{2} c_{3} Z+b_{1} c_{3} Z\right) \cos ^{2} \theta+\frac{3}{4} R\left(a_{1} c_{1}-b_{2} c_{1}-a_{2} c_{2}-b_{1} c_{2}+c_{2} Z\right. \\
& \left.+a_{10} \alpha+a_{3} Z \alpha-b_{10} \beta-b_{3} Z \beta-a_{10} \gamma-a_{3} Z \gamma\right) \sin \theta \cos ^{2} \theta \\
& -\frac{1}{2} R^{2}\left(a_{1} \alpha-b_{2} \alpha-a_{2} \beta-b_{1} \beta+Z \beta-a_{1} \gamma+b_{2} \gamma\right) \sin ^{3} \theta \cos \theta \\
& +\frac{3}{4} R\left(a_{2} c_{1}+b_{1} c_{1}-Z c_{1}+a_{1} c_{2}-b_{2} c_{2}+b_{10} \alpha+b_{3} Z \alpha+a_{10} \beta\right. \\
& \left.+a_{3} Z \beta-b_{10} \gamma-b_{3} Z \gamma\right) \sin ^{2} \theta \cos \theta+\frac{1}{4 R}\left(a_{2} c_{1} R^{2}-3 b_{1} c_{1} R^{2}\right. \\
& -4 c_{11} R^{2}+a_{1} c_{2} R^{2}-b_{2} c_{2} R^{2}+3 c_{1} Z R^{2}-4 c_{6} Z R^{2} \\
& -3 b_{10} \alpha R^{2}-3 b_{3} Z \alpha R^{2}+a_{10} \beta R^{2}+a_{3} Z \beta R^{2}-b_{10} \gamma R^{2}-b_{3} Z \gamma R^{2} \\
& \left.-4 b_{3} c_{3} Z^{2}\right) \cos \theta-\frac{1}{2}\left(2 c_{5} R^{2}-a_{1} \alpha R^{2}+b_{2} \alpha R^{2}-a_{2} \beta R^{2}+b_{1} \beta R^{2}\right. \\
& -Z \beta R^{2}-a_{1} \gamma R^{2}+b_{2} \gamma R^{2}-2 a_{3} c_{1} Z+2 b_{3} c_{2} Z-2 a_{1} c_{3} Z \\
& \left.+2 b_{2} c_{3} Z\right) \sin \theta \cos \theta-\frac{1}{8} R^{2}\left(a_{2} \alpha+b_{1} \alpha-Z \alpha+a_{1} \beta-b_{2} \beta\right. \\
& \left.-a_{2} \gamma-b_{1} \gamma+Z \gamma\right) \sin ^{4} \theta-\frac{1}{4} R\left(a_{1} c_{1}-b_{2} c_{1}-a_{2} c_{2}-b_{1} c_{2}+c_{2} Z\right. \\
& \left.+a_{10} \alpha+a_{3} Z \alpha-b_{10} \beta-b_{3} Z \beta-a_{10} \gamma-a_{3} Z \gamma\right) \sin ^{3} \theta-\frac{1}{2}\left(c_{4}+c_{7}\right) R^{2} \\
& +\frac{1}{2}\left(c_{3}-2 c_{9}\right) Z^{2}+\frac{1}{2}\left(c_{4} R^{2}-c_{7} R^{2}+b_{1} \alpha R^{2}-Z \alpha R^{2}+a_{2} \gamma R^{2}\right. \\
& \left.-c_{3} Z^{2}+b_{3} c_{1} Z-c_{10} Z+a_{3} c_{2} Z+a_{2} c_{3} Z+b_{1} c_{3} Z\right) \sin ^{2} \theta-\frac{1}{2}\left(b_{3} c_{1}\right. \\
& \left.-c_{10}+2 c_{13}-a_{3} c_{2}-a_{2} c_{3}+b_{1} c_{3}\right) Z+\frac{1}{8}\left(a_{2}-3 b_{1}\right) R^{2} \alpha+\frac{1}{8} R^{2} Z(3 \alpha+\gamma) \\
& +\frac{1}{8} R^{2}\left(a_{1} \beta-b_{2} \beta+3 a_{2} \gamma-b_{1} \gamma\right)+\frac{1}{4 R}\left(a_{1} c_{1} R^{2}-b_{2} c_{1} R^{2}-4 c_{12} R^{2}\right. \\
& +3 a_{2} c_{2} R^{2}-b_{1} c_{2} R^{2}+c_{2} Z R^{2}-4 c_{8} Z R^{2}+a_{10} \alpha R^{2}+a_{3} Z \alpha R^{2} \\
& \left.-b_{10} \beta R^{2}-b_{3} Z \beta R^{2}+3 a_{10} \gamma R^{2}+3 a_{3} Z \gamma R^{2}+4 a_{3} c_{3} Z^{2}\right) \sin \theta \text {. }
\end{aligned}
$$


Now the differential system (15) is in the normal formal for applying the averaging theory described in Theorem 11 of the appendix. Taking

$$
\mathbf{x}=(R, Z), \quad t=\theta, \quad T=2 \pi, \quad F_{1}(t, \mathbf{x})=\left(F_{11}(t, \mathbf{x}), F_{12}(t, \mathbf{x})\right)^{T},
$$

and

$$
F_{2}(t, \mathbf{x})=\left(F_{21}(t, \mathbf{x}), F_{22}(t, \mathbf{x})\right)^{T}, \quad \varepsilon^{3} R(t, \mathbf{x}, \varepsilon)=O\left(\varepsilon^{3}\right) .
$$

Note that here $D=\left[R_{1}, R_{2}\right] \times\left[Z_{1}, Z_{2}\right]$ where $0<R_{1}<R_{2}$ and $Z_{1}<0<Z_{2}$ with $R_{1}$ arbitrarily small and $R_{2},\left|Z_{1}\right|, Z_{2}$ arbitrarily large. So, system (15) satisfies the assumptions of Theorem 11, and it has the form (16). As $R \neq 0$ the functions $F_{1}$ and $F_{2}$ are analytical. Furthermore they are $2 \pi$-periodic in the variable $\theta$. Computing the averaging theory of first order we get that the average functions of $F_{1}$ are $f_{1}(\mathbf{x})=$ $\left(f_{11}(\mathbf{x}), f_{12}(\mathbf{x})\right)=\left(f_{11}(R, Z), f_{12}(R, Z)\right)$ with

$$
f_{11}(R, Z)=-\frac{1}{2}\left(a_{1}+b_{2}\right) R, \quad f_{12}(R, Z)=-c_{10}-c_{3} Z-\frac{1}{2} R^{2}(\alpha+\beta) .
$$

We have to find the zeros $\left(R^{*}, Z^{*}\right)$ of $f_{1}(R, Z)$, and to check that the Jacobian determinant $\left|D_{R, Z} f_{1}\left(R^{*}, Z^{*}\right)\right| \neq 0$. Solving the equation $f_{1}(R, Z)=0$ we obtain one solution

$$
\left(R^{*}, Z^{*}\right)=\left(0,-\frac{c_{10}}{c_{3}}\right)
$$

and

$$
\left|D_{R, Z} f_{1}\left(R^{*}, Z^{*}\right)\right|=\frac{c_{3}}{2}\left(a_{1}+b_{2}\right) .
$$

Hence, this solution provides a limit cycle bifurcating from the origin when $c_{3} c_{10}\left(a_{1}+\right.$ $\left.a_{2}\right) \neq 0$. Applying Theorem 11 and going back through the changes of variables we get the expression for the periodic solution given in the statement of Theorem 2 (a). This completes the proof of Theorem 2(a).

Now we set $f_{1} \equiv 0$ and we proceed to compute the function $f_{2}$ by applying the second order averaging theory. For that we take

$$
b_{2}=-a_{1}, \quad c_{3}=c_{10}=0 \quad \text { and } \quad \gamma=-\alpha .
$$

We have that $f_{2}$ is defined by

$$
f_{2}(R, Z)=\int_{0}^{2 \pi}\left[D_{R Z} F_{1}(\theta, R, Z) \cdot y_{1}(\theta, R, Z)+F_{2}(\theta, R, Z)\right] d \theta
$$

where

$$
y_{1}(\theta, R, Z)=\left(\int_{0}^{\theta} F_{11}(t, R, Z) d t, \int_{0}^{\theta} F_{12}(t, R, \mathbb{Z}) d t\right) .
$$

Moreover the Jacobian matrix is

$$
D_{R Z} F_{1}(\theta, R, Z)=\left(\begin{array}{cc}
\frac{\partial F_{11}}{\partial R} & \frac{\partial F_{11}}{\partial Z} \\
\frac{\partial F_{12}}{\partial R} & \frac{\partial F_{12}}{\partial Z}
\end{array}\right) .
$$

Doing so, we get $f_{2}(R, Z)=\left(f_{21}(R, Z), f_{22}(R, Z)\right)^{T}$, where

$$
\begin{aligned}
f_{21}(R, Z)= & -\frac{\alpha R^{3}}{4}-\left(a_{11}+b_{12}+a_{3} c_{2}-b_{3} c_{1}+\left(a_{6}+b_{8}\right) Z\right) R \\
f_{22}(R, Z)= & -\left(c_{4}+c_{7}+\left(a_{2}+b_{1}-Z\right) \alpha-a_{1} \beta\right) R^{2}-b_{10} c_{1}+a_{10} c_{2} \\
& -2 Z\left(b_{3} c_{1}-a_{3} c_{2}+c_{13}+c_{9} Z\right) .
\end{aligned}
$$


We have to find the zeros $\left(R^{*}, Z^{*}\right)$ of $f_{2}(R, Z)$, and to check that the Jacobian determinant $\left|D_{R, Z} f_{2}\left(R^{*}, Z^{*}\right)\right| \neq 0$. Solving the equation $f_{2}(R, Z)=0$ we obtain four solutions $\left(R^{*}, Z^{*}\right)$ which are:

$$
s_{1,2}=\left(0, \frac{-b_{3} c_{1}-c_{13}+a_{3} c_{2} \pm \sqrt{S_{13}}}{2 c_{9}}\right),
$$

with $S_{13}$ introduced in (6) and

$s_{3,4}=\left(\sqrt{-\frac{B}{2 A} \pm \frac{\sqrt{B^{2}-4 A C}}{2 A}}, \frac{\left(B \mp \sqrt{B^{2}-4 A C}\right) \alpha-8 A\left(a_{11}+b_{12}-b_{3} c_{1}+a_{3} c_{2}\right)}{8 A\left(a_{6}+b_{8}\right)}\right)$,

where $A, B, C$ were given in (7).

The Jacobian determinants for $s_{1,2}$ are

$d_{1,2}=\frac{\left(a_{6}+b_{8}\right) S_{13}}{c_{9}} \pm \sqrt{S_{13}}\left(\frac{-\left(a_{6}+b_{8}\right)\left(b_{3} c_{1}+c_{13}-a_{3} c_{2}\right)}{c_{9}}+2\left(a_{11}+b_{12}-b_{3} c_{1}+a_{3} c_{2}\right)\right)$,

respectively. Thus, the solutions $s_{1,2}$ provide a limit cycle bifurcating from the origin when $S_{13}>0, c_{9} \neq 0$ and $d_{1,2} \neq 0$.

The Jacobian determinants for $s_{3,4}$ are

$$
\begin{aligned}
d_{3,4}= & \frac{1}{4 A^{2}\left(a_{6}+b_{8}\right)}\left(B\left(\sqrt{B^{2}-4 A C}-B\right)\left(2\left(a_{6}+b_{8}\right)+c_{9}\right) \alpha^{2}\right. \\
& +2 A\left(-2\left(\sqrt{B^{2}-4 A C}-B\right)\left(c_{4}+c_{7}+\left(a_{2}+b_{1}\right) \alpha-a_{1} \beta\right) a_{6}^{2}\right. \\
& +\left(\left(\alpha\left(\sqrt{B^{2}-4 A C}\left(-2 a_{11}-2 b_{12}+3 b_{3} c_{1}+c_{13}-3 a_{3} c_{2}\right)+2 C \alpha\right)\right.\right. \\
& -4 b_{8} \sqrt{B^{2}-4 A C}\left(c_{4}+c_{7}+\left(a_{2}+b_{1}\right) \alpha-a_{1} \beta\right)+B\left(2\left(a_{11}+b_{12}\right) \alpha\right. \\
& \left.\left.-\left(3 b_{3} c_{1}+c_{13}-3 a_{3} c_{2}\right) \alpha+4 b_{8}\left(c_{4}+c_{7}+\left(a_{2}+b_{1}\right) \alpha-a_{1} \beta\right)\right)\right) a_{6} \\
& +c_{9} \alpha\left(C \alpha-2 \sqrt{B^{2}-4 A C}\left(a_{11}+b_{12}-b_{3} c_{1}+a_{3} c_{2}\right)\right) \\
& +b_{8} \alpha\left(\sqrt{B^{2}-4 A C}\left(-2 a_{11}-2 b_{12}+3 b_{3} c_{1}+c_{13}-3 a_{3} c_{2}\right)+2 C \alpha\right) \\
& -2 b_{8}^{2} \sqrt{B^{2}-4 A C}\left(c_{4}+c_{7}+\left(a_{2}+b_{1}\right) \alpha-a_{1} \beta\right)+B\left(2 \left(c_{4}+c_{7}\right.\right. \\
& \left.+\left(a_{2}+b_{1}\right) \alpha-a_{1} \beta\right) b_{8}^{2}+\left(2 a_{11}+2 b_{12}-3 b_{3} c_{1}-c_{13}+3 a_{3} c_{2}\right) \alpha b_{8} \\
& \left.\left.\left.+2\left(a_{11}+b_{12}-b_{3} c_{1}+a_{3} c_{2}\right) c_{9} \alpha\right)\right)\right) .
\end{aligned}
$$

Thus the solutions $s_{3,4}$ provide two limit cycles bifurcating from the origin when

$$
C \neq 0,-\frac{B}{2 A}+\frac{\sqrt{B^{2}-4 A C}}{2 A}>0 \quad \text { and } \quad d_{3,4} \neq 0 .
$$

Applying Theorem 11 and going back through the changes of variables we get the expression for the periodic solution given in the statement of Theorem 2(b). This completes the proof of Theorem 2. 


\section{Appendix: Periodic orbits VIA the AVERAging theory of FIRST AND SECOND ORDER}

In this section we recall the averaging theory of second order to find periodic orbits. The averaging theory up to third order specifically for studying periodic orbits was developed in $[1,12]$, see the former paper for additional details and for the proofs of the results stated in this section related with the averaging theory.

In this work we only need this theory up to second order. It is summarized as follows.

Theorem 11. Consider the differential system

$$
\dot{x}(t)=\varepsilon F_{1}(t, x)+\varepsilon^{2} F_{2}(t, x)+\varepsilon^{3} R(t, x, \varepsilon),
$$

where $F_{1}, F_{2}: \mathbb{R} \times D \rightarrow \mathbb{R}^{n}, R: \mathbb{R} \times D \times\left(-\varepsilon_{f}, \varepsilon_{f}\right) \rightarrow \mathbb{R}^{n}$ are continuous functions, $T$-periodic in the first variable, and $D$ is an open subset of $\mathbb{R}^{n}$. Assume that the following hypothesis (i) and (ii) hold.

(i) $F_{1}(t, \cdot) \in C^{1}(D)$ for all $t \in \mathbb{R}, F_{1}, F_{2}, R$ and $D_{x} F_{1}$ are locally Lipschitz with respect to $x$, and $R$ is differentiable with respect to $\varepsilon$. We define $f_{1}, f_{2}$ : $D \rightarrow \mathbb{R}^{n}$ as

$$
\begin{aligned}
& f_{1}(z)=\int_{0}^{T} F_{1}(s, z) d s \\
& f_{2}(z)=\int_{0}^{T}\left[D_{z} F_{1}(s, z) \int_{0}^{s} F_{1}(t, z) d t+F_{2}(s, z)\right] d s .
\end{aligned}
$$

(ii) For $V \subset D$ an open and bounded set and for each $\varepsilon \in\left(-\varepsilon_{f}, \varepsilon_{f}\right) \backslash\{0\}$, there exist $a \in V$ such that $f_{1}(a)+\varepsilon f_{2}(a)=0$ and $d_{B}\left(f_{1}+\varepsilon f_{2}\right) \neq 0$ (see its definition later on).

Then for $|\varepsilon|>0$ sufficiently small, there exists a $T$-periodic solution $\varphi(\cdot, \varepsilon)$ of the system such that $\varphi(0, \varepsilon) \rightarrow$ a when $\varepsilon \rightarrow 0$.

As usual we have denoted by $d_{B}\left(f_{1}+\varepsilon f_{2}\right)$, the Brouwer degree of the function $f_{1}+\varepsilon f_{2}: V \rightarrow \mathbb{R}^{n}$ at its fixed point $a$. A sufficient condition for showing that the Brouwer degree of a function $f$ at its fixed point $a$ is non-zero, is that the Jacobian of the function $f$ at $a$ (when it is defined) is non-zero, see for instance [9].

If the function $f_{1}$ is not identically zero, then the zeros of $f_{1}+\varepsilon f_{2}$ are approximately the zeros of $f_{1}$ for $\varepsilon$ sufficiently small. In this case Theorem 11 provides the so-called averaging theory of first order.

If the function $f_{1}$ is identically zero and $f_{2}$ is not identically zero, then the zeros of $f_{1}+\varepsilon f_{2}$ are the zeros of $f_{2}$. In this case Theorem 11 provides the so-called averaging theory of second order.

The method based on Theorem 11 consists essentially of finding $T$-periodic solutions for a differential system whose vector field depends on a small parameter $\varepsilon$, by means of the averaging method. A quantitative relation between the solutions of some non-autonomous periodic differential system and the solutions of the averaged differential system, which is autonomous, is obtained. In this way a finite dimensional function related with the original problem is found, the simple zeros of this function correspond to the periodic orbits of the non-autonomous periodic differential system for values of the parameter $\varepsilon$ different from zero and sufficiently 
small. Here a simple zero $a$ of a function $f$ means that the Jacobian of $f$ at $a$ is not zero.

\section{ACKNOWLEDGEMENTS}

The first and third authors were supported by Portuguese National Funds through FCT - Fundação para a Ciência e a Tecnologia within the projects PTDC/MAT /117106/2010 and PEst-OE/EEI/LA0009/2013 (CAMGSD).

The second author is partially supported by a MINECO/FEDER grant MTM200803437 and MTM2013-40998-P, an AGAUR grant number 2013SGR-568, an ICREA Academia, the grants FP7-PEOPLE-2012-IRSES 318999 and 316338, FEDER-UNAB104E-378, and a CAPES grant number 88881.030454/2013-01 from the program CSFPVE.

\section{REFERENCES}

[1] A. Buică, J. Giné And J. Llibre, A second order analysis of the periodic solutions for nonlinear periodic differential systems with a small parameter, Physica D 241 (2012), 528-533.

[2] C. Christopher, J. Llibre And J.V. Pereira, Multiplicity of invariant algebraic curves in polynomial vector fields, Pacific J. Math. 229 (2007), 63-117.

[3] G. Darboux, Mèmoire sur les équations différentielles algébraiques du premier ordre et du premier degré (Métanges), Bull. Sci. Math. 2éme série 2 (1878), 60-96; 123-144; 151-200.

[4] F. Dumortier, J. Llibre And J.C. Artés, Qualitative theory of planar differential systems, Universitext, Springer-Verlag, New York, 2006.

[5] Y.A. Kuznetsov, Elements of Applied Bifurcation Theory, third ed., Springer-Verlag, NewYork, 2004.

[6] G.X. LI, R.H. RAND AND F.C. Moon, Bifurcations and chaos in a forced zero-stiffness impact oscillator, Internat. J. Non-Linear Mech. 25 (1990), 417-432.

[7] J. Llibre And X. Zhang, On the Darboux integrability of the polynomial differential systems, Qualitative Theory and Dynamical Systems 11 (2012), 129-144.

[8] J. Llibre And X. Zhang, Darboux theory of integrability for polynomial vector fields in $\mathbb{R}^{n}$ taking into account the multiplicity at infinity, Bull. Sci. Math. 133 (2009), 765-778.

[9] N. G. Lloyd, Degree Theory, Cambridge Tracts in Mathematics, Vol. 73 Cambridge University Press, 1978

[10] A. Mahdi, V.G. Romanovski and D.S. Shafer, Stability and periodic oscillations in the Moon-Rand systems, Nonlinear Analysis: Real World Applications 14 (2013), 294-313.

[11] F.C. Moon And R.H. Rand, Parametric stiffness control of flexible structures, in Jet Propulsion Laboratory Publication 85-29, vol. II, California Institute of Technology, 1985, pp. 329-342.

[12] F. Verhulst, Nonlinear Differential Equations and Dynamical Systems, Universitext Springer Verlag, 1996.

${ }^{1}$ Departamento de Matemática, Instituto Superior Técnico, Universidade de Lisboa, Av. Rovisco Pais 1049-001, Lisboa, Portugal

E-mail address: barreira@math.ist.utl.pt

2 Departament de Matemàtiques, Universitat Autònoma de Barcelona, 08193 Bellaterra, Barcelona, Catalonia, Spain

E-mail address: jllibre@mat.uab.cat

${ }^{3}$ Departamento de Matemática, Instituto Superior Técnico, Universidade de Lisboa,

Av. Rovisco Pais 1049-001, Lisboa, Portugal

E-mail address: cvalls@math.ist.utl.pt 